\title{
Feline coronavirus infection
}

\author{
Aleksey Ermakov ${ }^{1}$, Tatyana Lipilkina ${ }^{1}$, Pavel Lipilkin ${ }^{1}$ and Igor Popov $^{1 *}$ \\ ${ }^{1}$ Don state technical university, Gagarin Square, 1, Rostov-on-Don, 344000, Russia
}

\begin{abstract}
The main feature of feline coronavirus infection is its manifestation in the form of peritonitis. Feline infectious peritonitis is a highly lethal disease that lacks primary prevention and therapy. Therefore, feline infectious peritonitis is an epizootic problem in the near future. In our review, we demonstrate the current clinical, diagnostic, and therapeutic interventions for feline infectious peritonitis, as well as hypotheses of origin.
\end{abstract}

\section{Feline infectious peritonitis (FIP)}

Feline infectious peritonitis (FIP) is a disease of domestic and feral cats worldwide. The causative agents of FIP belong to the genus Alphacoronavirus [1]. FIP was first officially described in the feline population in 1963 at Angell Animal Medical Center Hospital, Boston [2]. The etiologic agent of feline coronavirus (FCoV) occurs in two serotypes, which can be distinguished by their biological behavior but not by their morphology. Both serotypes are capable of causing FIP [3], serotype I being more common in nature and responsible for $80-90 \%$ of natural clinical cases $[4,5]$. Serotype II is relatively rare and resulted from recombination between cat coronavirus serotype I and canine intestinal coronavirus (CCoV) serotype II following interspecies transmission to cats (The transmission lines are shown in Figure 1).

Interspecies transmission of canine intestinal coronavirus to cats resulting in genetic recombination between serotype I FECV and serotype II CCoV resulted in serotype II FECV $[6,7,8]$.

\footnotetext{
* Corresponding author : doc.igor.popov@gmail.com
} 


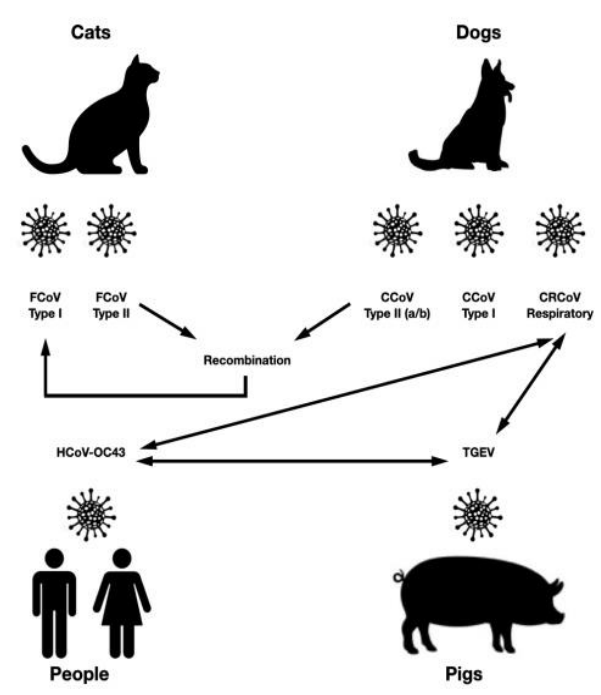

Fig. 1. Possible transmission routes of genetically related CoVs. FCoV type I virus has a feline spike protein, whereas type II FCoV spike protein is recombinant between FCoV and CCoV type II. Antigenic similarities between animal and human CoV, namely TGEV, human HCoV-OC43 and CIRD, were established by ELISA.

\section{Origins}

The registration of FIP cases in the late 1950s was documented by records at Boston's Angell Animal Medical Center [9]. There was a steady increase in incidence in the 1960s, and FIP is now one of the leading infectious causes of death among young shelter and kennel cats. The reason for the sudden emergence of FIP is unknown, but there are at least three hypotheses.

First, FIP began to be mentioned after the first descriptions of TGEV transmissible gastroenteritis of pigs in North America [10]. FIP virus is closely related to TGEV and $\mathrm{CCoV}$ [11]. Recombinants are known to occur between these three viruses [12, 13, 14]. At least one $\mathrm{CCoV}$ strain can cause enteritis in cats and amplify subsequent $\mathrm{CCoV}$ infection [15]. Therefore, CCoV may be a probable precursor of FECV. Recombination events are favored by the ease with which transcription units (RNA) can be obtained or lost during the different evolution of coronaviruses [16].

Second, the FIP mutation may be selective with respect to the FIP variant that appeared in the 1950s. This variant could also have arisen due to intra- and interspecies mutation of coronaviruses in general and, in this case, $\mathrm{FCoV}$ in particular.

A third explanation could include changes in the fact that the second half of the 20th century saw a shift in the status, keeping and breeding of cats as pets. The number of pets increased, pure breeding and kennel breeding became more and more popular and more cats, and in particular kittens, ended up in shelters. Cats were known to become infected with enteric coronavirus (FECV) and FIP in these many feline facilities.

Using the ELISA method, the antigenic association of FIP with other human and animal coronaviruses has been studied. These studies demonstrate that FIP virus has antigenic similarity with known coronaviruses, namely TGEV, human HCoV-OC43, 229E virus, and $\operatorname{CCoV}[17,18,19,20]$.

Similar to the interspecies transmission of coronavirus from dogs to cats, recombination between $\mathrm{CCoV}$ serotype II and other coronaviruses occurred, which led to the appearance 
of canine coronavirus variants with $\mathrm{N}$-terminal spike protein domains homologous to TGEV [21]. Canine respiratory coronavirus $(\mathrm{CRCoV})$ is an important etiological component of canine infectious respiratory disease (CIRD) [22]. CIRD is a highly contagious respiratory disease syndrome and is easily transmitted by aerosol transmission between dogs [23].

\section{Epizootiology and clinic}

Feline intestinal coronavirus (FECV) is highly virulent and is transmitted by the fecal-oral route. But most often the infection is asymptomatic or causes only mild and transient diarrhea $[24,25,26]$. Feline infectious peritonitis virus (FIPV), on the other hand, is not transmitted by the fecal-oral route, but results from mutation of avirulent FECV of infected cats and then causes highly lethal FIP [27, 28, 29]. Coronaviruses are capable of causing disease in humans by crossing interspecies barriers, including from companion animals [30].

Initially, FECV enters the gastrointestinal tract of the cat through the nasopharynx. Several variants of the course of the disease are then possible:

- $\quad$ elimination of the virus without the development of disease;

- $\quad$ asymptomatic carrier;

- $\quad$ FECV development;

- $\quad$ mutation FECV to FIPV [31, 32].

The pathogenesis of FIPV development has 2 pathways:

1.elimination of the virus without the development of disease;

2.presence of FIPV, resulting in viremia and persistence of the virus in macrophages. Later virus replication occurs in perivascular tissues with formation of AG-AT complex. Accumulation of active complexes in the walls of small blood vessels, complement and coagulation cascades activation at the very last stage occurs development of immunemediated vasculitis and perivasculitis [33].

Clinically, FIP manifests itself in three forms:

- effusive (wet, non-parenchymatous). Transmissible inflammatory state with exudation into the abdominal cavity [34].

- granulomatous (dry, parenchymatous). Parenchymatous organ and central nervous system (CNS) involvement [35, 36, 37].

- $\quad$ asymptomatic carrier [38].

The effusive form of FIP is the most common. Two forms manifest themselves simultaneously quite rarely, more often it is a transient form of dry to wet or vice versa. The incubation period of FIP is from 2 to 14 days [39].

The effusive form of FIP is characterized by respiratory changes and respiratory failure syndrome [40]. In the dry form of FIP, CNS involvement predominates in $60 \%$ of cats. Signs associated with the abdominal cavity are observed in $40 \%$ of animals. Involvement of the wall of the cecum and colon is a specific form of dry peritonitis associated with signs of typical ulcerative colitis [41].

Clinical signs seen in both effusive and non-effusive FIP include lethargy, anorexia, weight loss (or failure to gain weight/stunted growth in younger cats), a fluctuating pyrexia that is usually non-responsive to drugs such as antibiotics or non-steroidal antiinflammatories, and sometimes jaundice (more common in effusive FIP). FIP.

Lymphadenomegaly can also be present in both the effusive and non-effusive forms of

Asymptomatic FCoV infection was thought to be confined to the intestinal tract, but we now know that clinically healthy cats infected with FCoV may have systemic infection with a lower viral load than cats with FIP. 
It is important to remember that clinical signs of FIP can change over time, so repeated clinical examinations are important to detect newly apparent signs (eg, development of a small volume of effusion, ocular changes visible on retinal examination).

Risk factors for FIP [42, 43]:

- $\quad$ early age of cats (under 2 years of age);

- pedigree cats;

- $\quad$ presence of comorbidities;

- $\quad$ keeping a large number of cats in one place;

- frequent visits to veterinary clinics.

The need to study coronavirus infection in companion animals stems from the fact that the virus frequently mutates and is capable of crossing interspecies barriers between animals and humans. Currently, there are no confirmed cases of coronavirus transmission from cats to humans, but a close relationship of $\mathrm{CoV}$ with bovine $\mathrm{CoV}$ and $\mathrm{HCoV}-\mathrm{OC} 43$ was established, which also indicates one common precursor virus, which does not exclude the possibility of transmission. It is also related, with the COVID-19 pandemic beginning in 2019. Cats are the most informative experimental model of this disease for evaluating antiviral drugs or candidate vaccines against SARS-CoV-2.

In cats, coronavirus is transmitted by aerosol transmission only under experimental conditions when SARS-CoV-2 is inoculated. These results indicate that SARS-CoV-2 can replicate effectively in cats and that it is possible that the virus can be transmitted from cats by the aerosol route [44].

Thus, the urban environment and veterinary medicalization are generalized conditions for the preservation of an epizootic situation, which creates a precedent for creating other preventive strategies and taking into account in such strategies the interspecies transfer of coronaviruses. The role of asymptomatic cases in the spread of FIP is still unclear

\section{Diagnosis of FIP}

Haematological changes in FIP are non-specific, but there are a number of abnormalities that can be looked for to support a diagnosis. Lymphopenia is particularly common (55$77 \%$ of cases, although a recent study found only $49.5 \%$ of FIP cases to be lymphopenic), with neutrophilia (39-57\%), a left shift, and mild to moderate normocytic, normochromic anaemia (37-54\%) also reported. An association between FIP and microcytosis (with or without anaemia) was recently reported. Severe immune-mediated haemolytic anaemia (IMHA), with an associated regenerative anaemia, can occur with FIP, but is uncommon.

The changes in serum biochemistry in FIP cases are varied and often non-specific, but there are a number of important abnormalities that should be looked for to support a diagnosis of FIP.

Hyperglobulinaemia is reported in $89 \%$ of cases, often with hypoalbuminaemia or lownormal serum albumin (seen in $64.5 \%$ of cases). Hyperbilirubinaemia occurs in $21-63 \%$ of FIP cases, and is especially seen with effusive FIP, often without marked elevations in alanine aminotransferase (ALT), alkaline phosphatase (ALP) or $\gamma$-glutamyltransferase enzyme activity (although these can be moderately elevated in FIP cases).

In the absence of a definitive diagnosis, a high index of suspicion of FIP may be obtained from background information, clinical signs and routine clinicopathological results.

In vivo diagnosis of FIP is difficult. No molecular genetic or serologic tests can differentiate between FIPV and FECV. Consequently, real-time PCR (qPCR) or serology can confirm infection but not disease. FIP can be misdiagnosed in cats with positive qPCR with nonspecific clinical signs because of diseases other than FIP. Conversely, cats with FIP may become qPCR negative or seronegative $[45,46]$. 
Ultimately, only the detection of virus in the sweat can confirm the disease, although some clinical or clinicopathological changes may also be indicative of FIP [47, 48]. The organs with the highest viral load include the omentum, mesenteric lymph nodes and spleen, so these tissues are most useful for analysis by qPCR [49]. This method is rarely applied to tissue samples for FIP diagnosis in veterinary practice because it requires invasive collection of tissue samples by laparotomy or laparoscopy or can be done postmortem. Thus, this study is mainly for research purposes and is not used as a clinical diagnostic test for FIP [50].

Serum antibody test is carried out in the form of ELISA or rapid immunomigration tests [51]. Most use CoV-infected swine or feline cells as a substrate and titres are read in distinct multiples of serum dilutions. A positive test for antibodies to FCoV indicates that the cat is infected with $\mathrm{FCoV}$ and seroconverts (this takes 2-3 weeks from infection). Differences in mean anti-FCoV antibody titer were found across cat breeds, which may reflect differences in breed response to FCoV infection [52]. FIP cats have higher FCoV antibody titers than non-FIP cats, but there are cases with no difference between median FCoV antibody titers in healthy cats and cats with FIP. Thus, an individual cat's antibody titer is of limited value for FIP verification [53]. Some clinically healthy cats have falsepositive FCoV antibody titers, while about $10 \%$ of cats with FIP are seronegative [54] indicating difficulty in interpretation. However, false negative results have also been reported in FIP cases [55].

Analyzing an effusion sample when FIP is suspected is useful for diagnosis. Ultrasonography is considered more sensitive than X-ray to detect small pockets of fluid in the chest and abdomen. Ultrasonography is recommended for sampling any small volume of effusion, and similarly, can be used to sample small pockets of fluid [56]. ELISA and qPCR for FCoV RNA on effusion samples can also be performed.

In cats with wet FIP, a laboratory fluid test, including the Rivalt is a crude point-of-care assay that can be performed on an effusion sample to allow rapid differentiation of transudate from exudate. If this test is negative, the possibility of FIP is very unlikely. However, if the Rivalt test is positive, further diagnostic steps should be performed. FIP effusions are usually clear, viscous, straw yellow, and rich in protein (eosinophilia is described by cytology) with a protein concentration of $>35 \mathrm{~g} / 1[57,58]$.

Magnetic resonance imaging in cases with neurological clinical signs, imaging of the brain by MRI may be useful to demonstrate changes. For example, obstructive hydrocephalus, syringomyelia, foramen magnum herniation and marked contrast enhancement of the meninges, third ventricle, mesencephalic aqueduct and brainstem have been reported with FIP [59].

Cerebrospinal fluid (CSF) can be collected from neurological cases, although care should be taken as the risk of brain herniation is significant. CSF may show elevated protein concentrations $(>0.30 \mathrm{~g} / 1$ cisternal samples, $>0.46 \mathrm{~g} / \mathrm{l}$ lumbar samples; occasionally FIP cats show marked elevations of $>2 \mathrm{~g} / \mathrm{l})$ and an increased cell count $(>8 \times 106$ cells/1 lumbar and cisternal samples; FIP cats can have counts of $>1000 \times 106$ cells/l), with the cell type being predominantly neutrophilic, mononuclear or mixed [60]. Some neurological cases of FIP have unremarkable CSF analysis results. Samples of CSF can also be submitted for qPCR for FCoV RNA and immunostaining for FCoV antigen (see below).

Key points that may indicate a history of FIP and on clinical examination:

- $\quad$ a young cat from a family (including shelters or catteries);

- neurological signs;

- $\quad$ find and take samples of pleural or peritoneal effusion. The effusions are usually clear, viscous, and straw-yellow visually, with a protein concentration $>35 \mathrm{~g} / \mathrm{l}$. FIP effusions are small-celled (usually $<5 \times 109$ cells/l).

Be aware of the clinical value of the tests available for the diagnosis of FIP: 
- ELISA;

- Detection of FCoV RNA by qPCR, especially if present at high levels, in diagnostic specimens such as effusions, cerebrospinal fluid and biopsies.

However, any diagnostic procedures must be agreed with the cat's owner. Especially the veterinary community must recognize that today all laboratory diagnostics does not alter clinical outcomes, no matter how much they are performed. A balance must be struck between diagnostic capabilities and clinically relevant action by the veterinarian.

\section{Treatment and prevention of FIP}

Treatment of FIP until recently has focused on maintenance therapy and stimulation of cellmediated immunity with interferon or feline recombinant interferon [61].

In the last few years, the peptidomimetic GC-376 and the nucleoside analogue GS441524 have been developed, both capable of inhibiting FCoV replication in various ways. They have been tested in vitro, in experimental and natural FIP infections in cats $[62,63$, 64]. GC-376 induced remission of the disease in $35 \%$ of treated cats and was more effective against certain clinical manifestations of FIP [65]. In turn, GS-441524 significantly reduced the viral load in infected cats and induced remission of clinical signs after one or more cycles of treatment in $96.1 \%$ of cats. But GS-441524 is not registered to treat FIP in cats in many countries. And treatment with unlicensed drugs is not safe [66].

Currently, one live virus-based vaccine against FIP (intranasal) has been developed and commercialized and is available in only a few countries [67]. It should be administered to kittens 16 weeks of age or older. According to the World Small Animal Veterinary Association (WSAVA), FIP vaccine is not recommended [68].

The key difficulty of many preventive measures is the complexity of their implementation within the framework of the existing contradictions between government and business strategies to improve the epizootic situation. The state veterinary expertise is aimed at ensuring food safety, and the veterinary business, which deals with the health of domestic animals, does not have the resources to ensure the prevention of the spread of coronavirus infections among live animals, including FCoV. At the same time, measures to prevent the spread of infectious diseases are not always followed in veterinary business practice.

However, we believe that the economic burden that cat owners bear is not commensurate with the preventive and clinical results that can be obtained at the current state of the art in veterinary medicine.

\section{Conclusion}

A diagnosis of FIP can be made based on clinical signs. This can be used to discuss with the owner about the need for additional invasive diagnostic tests (biopsy).

In such cases, euthanasia is discussed as an alternative to definitive diagnosis prior to death; this may be preferable, for example, for cats from shelters or when the cats are very sick and there is concern about the patient's ability to tolerate further diagnostic procedures. If euthanasia is performed without a definitive diagnosis, postmortem examination is strongly recommended.

Since there is no effective specific prophylaxis, the only method is to isolate sick animals and distance them until repeated qPCR tests give a negative result [69].

Thus, the high contagiousness and mortality of FIP, as well as the lack of effective diagnostic, therapeutic and preventive measures, lead to the need to comply with epizootiology measures from the standpoint of reducing the risks of the spread of FIP to 
other cats in veterinary clinics. The phenomenon of veterinary medicalization does not improve the epizootiology situation for coronavirus infections, not only among cats, but also creates a probable threat of the spread of coronavirus infection among other animals. But despite the developed ability of veterinarians to provide diagnostic capabilities, this is not reflected in the control of morbidity and the creation of high-quality FIP registries. The lack of proper veterinary control of the incidence of FIP is a factor in which proper preventive measures cannot be applied.

\section{Acknowledgements}

The reported study was funded by RFBR according to the research project № 20-04-60263.

\section{References}

1. J.M.A. Van den Brand, B.L. Haagmans, L. Leijten, et al., Vet. pathol 45, 551-562 (2008) doi.org/10.1354/vp.45-4-551

2. J.E. Holzworth, Cornell Vet, 53, 157-160 (1963)

3. M.R. Denison, R.L. Graham, E.F. Donaldson et al., RNA Biol 8, 270-271 (2011) doi:10.4161/rna.8.2.15013

4. L.M. Desmarets, B.L. Vermeulen, S. Theuns et al., Scientific Reports 6, 20022 (2016)

5. D. D. Addie, M. McDonald, S. Audhuy et al., J Feline Med Surg 14, 171-176 (2012) doi:10.1177/1098612X11429644

6. Y. Terada, N. Matsui, K. Noguchi et al., PLoS ONE 9, 106534 (2014) doi:10.1371/journal.pone.0106534

7. D. Addie, Infectious disease of the dog and cat (Elsevier, St Louis, MO, 2012)

8. J.A. Jaimes, G.R. Whittaker, Virology 9 (2018) doi:10.1016/j.virol.2017.12.027

9. E. N. Baker, S. Tasker, T.J. Gruffydd-Jones, J Vet Intern Med 27, 445-450 (2013)

10. Y. Kim, H. Liu, A. C. Galasiti-Kankanamalage et al., PLoS Pathog 12, 1005531 (2016) doi:10.1371/journal.ppat.1005531

11. S. Belouzard, J. K. Millet, B. N. Licitra et al., Viruses 6, 1011-1033 (2012) doi:10.3390/v4061011

12. A. Kipar, M. L. Meli, Vet Pathol, 51, 505-507 (2014) doi:10.1177/0300985814522077

13. L. Golovko, L. A. Lyons, H. Liu et al, Virus Res 175, 58-63 (2013) doi:10.1016/j.virusres.2013.04.006

14. N. C. Pedersen, H. Liu, M. Durden et al., Vet Immunol Immunopathol 171, 17-20 (2016) doi:10.1016/j.vetimm.2016.01.002

15. B. S. Bauer, M. E. Kerr, L. S. Sandmeyer et al., Vet Ophthalmol 1, 160-163 (2013) doi:10.1111/vop. 12044

16. H. Y. Tsai, L. L. Chueh, C. N. Lin et al., J Feline Med Surg 13, 74-80 (2011) doi:10.1016/j.jfms.2010.09.014

17. A. Stranieri, A. Giordano, S. Bo et al., J Feline Med Surg 8, 880-887 (2017) doi:10.1177/1098612X16664389

18. K. Hazuchova, S. Held, R. Neiger, J Feline Med Surg 8, 809-816 (2017) doi:10.1177/1098612X16658925

19. E. Lorusso, V. Mari, M. Losurdo et al., Res Vet Sci 125, 421-424 (2019) doi:10.1016/j.rvsc.2017.10.004 
20. E. N. Barker, A. Stranieri, C. R. Helps et al., Vet Res 48, 60 (2017) doi:10.1186s 13567-017-0467-9

21. A. Stranieri, S. Lauzi, A. Giordano et al., J Virol Methods 243, 105-108 (2017) doi:10.1016/j.jviromet.2017.01.009

22. L. Longstaff, E. Porter, V. J. Crossley et al., J Feline Med Surg 19, 240-245 (2017) doi:10.1177/1098612X15606957

23. J. A. Mitchell, H. W. Brooks, B. Szladovits et al., Vet. Microbiol 162, 582-594 (2013) doi:10.1016/j.vetmic.2012.11.025

24. S.J. Doenges, K. Weber, R. Dorsch et al., J Feline Med Surg 18, 104-109 (2016) doi:10.1177/1098612X15574757

25. S. Felten, K. Matiasek, S. Gruendl et al., J Feline Med Surg 19, 410-418 (2017) doi:10.1177/1098612X16630357

26. A. S. Abdel-Moneim, E. M. Abdelwhab, Pathogens 30, 529 (2020) doi:10.3390/pathogens 9070529

27. D. D. Addie, S. Curran, F. Bellini et al., Research in Veterinary Science 130, 222-229 (2020) doi: 10.1016/j.rvsc.2020.02.012

28. A. Balint, A. Farsang, L. Szeredi et al., Vet. Microbiology 169, 154-162 (2014) doi:10.1016/j.vetmic.2013.10.015

29. N. C. Pedersen, J. F. Boyle, K. Floyd et al., Am. J. Vet. Res 42, 368-377 (1981)

30. I. M. Donnik, Ig. V. Popov, S. V. Sereda, Il. V. Popov, M. L. Chikindas, A.M. Ermakov, Biology Bulletin 48, 26-30 (2021) doi:10.31857/S0002332921010057

31. A. Kipar, M.L. Meli, Vet Pathol 51, 515-526 (2014) doi: 10.1177/0300985814522077.

32. F. Riemer, K.A. Kuehner, S. Ritz et al., J. Feline Med. Surg 18, 354-356 (2016) doi:10.1177/1098612X15586209

33. D.D. Addie, Veterinary Nursing Journal 34(8), 201-206 (2019) doi:10.1080/17415349.2019.1629366

34. L. Giori, A. Giordano, C. Giudice et al., J. Small Anim. Pract 52, 152-157 (2011) doi:10.1111/j.1748-5827.2011.01042.x.

35. Y. Fischer, G. Wess, K. Hartmann, Schweizer Archiv Tierheilkunde 154, 27-31 (2012) doi:10.1024/0036-7281/a000289.

36. E.J. Ives, A.E. Vanhaesebrouck, F. Cian, J Feline Med Surg 15, 1149-1153 (2013) doi: 10.1177/1098612X13491960

37. N.C. Pedersen, The Veterinary Journal 201, 133-141 (2014) doi:10.1016/j.tvj1.2014.04.016

38. A. Giordano, A. Stranieri, G. Rossi, S. Paltrinieri, Vet. Clin. Pathol 44, 295-302 (2015) doi:10.1111/vcp.12241

39. M. B. D. Lowiese, T. Sebastiaan, A. J. O. Dominique et al., Veterinary Research 44, 113 (2013) doi:10.1186/1297-9716-44-71

40. L. W. Myrrha, F. M. F. Silva, E. F. Peternelli et al., Advances in Virology, 1-8 (2011) doi. org/10.1155/2011/109849

41. A. Stranieri, S. Paltrinieri, A. Giordano, J. Vet. Diagn. Investig 29, 321-324 (2017) doi:10.1177/1040638716689115

42. N. Ziolkowska, K. Pazdzior-Czapula, B. Lewczuk et al., Vet. Pathol 54, 933-944 (2017) doi:10.1177/0300985817728557 
43. F. Riemer, K. A. Kuehner, S. Ritz, et al., Journal of Feline Medicine and Surgery 18(4), 348-356 (2016) doi:10.1177/1098612X15586209

44. J. Shi, Z. Wen, G. Zhong et al., Science 368, 1016-1020 (2020) doi:10.1126/science.abb7015

45. D. D. Addie, S. le Poder, P. Burr et al., J. Feline Med. Surg 17, 152-162 (2015) doi:10.1177/1098612X14538873

46. M. L. Meli, P. Burr, N. Decaro et al., Journal of Feline Medicine and Surgery 15, 295 299 (2013) doi:10.1177/1098612X12467995

47. S. Felten, K. Hartmann, Viruses 11, 1068 (2020) doi:10.3390/v11111068

48. A. Stranieri, A. Giordano, S. Paltrinieri et al., Journal of Veterinary Diagnostic Investigation 30, 459-463 (2018) doi:10.1177/1040638718756460

49. S. Gruendl, K. Matiasek, L. Matiasek et al., J Feline Med Surg 19, 576-585 (2017) doi:10.1177/1098612X16640839

50. N. C. Pedersen, C. Eckstrand, H. Liu et al., Vet. Microbiol 175, 157-166 (2015) doi:10.1016/j.vetmic.2014.10.025

51. T. Soma, N. Saito, M. Kawaguchi et al., J. Vet. Med. Sci 80, 59-62 (2018) doi:10.1292/jvms.17-0399

52. D. R. Rissi, J. Vet. Diagn. Investig 30, 396-399 (2018) doi: $10.1177 / 1040638718755833$

53. S. Felten, K. Hartmann, S. Doerfelt et al., J. Vet. Diagn. Investig 31, 210-216 (2019) doi: $10.1177 / 1040638718825280$

54. M. L. Meli, P. Burr, N. Decaro, J Feline Med Surg 15, 295-299 (2013) doi:10.1177/1098612X12467995

55. N. Stephenson, P. Swift, R. B. Moeller et al., USA. J. Wildl. Dis 49, 408-412 (2013) doi: $10.7589 / 2012-08-210$

56. S. Tasker, J Feline Med Surg 20, 235 (2018) doi:10.1177/1098612X18758592

57. A.L. Litster, R. Pogranichniy, T.L. Lin, Vet. J. 198, 362-366 (2013) doi:10.1016/j.tvj1.2013.08.023

58. Y. Fischer, C. Sauter-Louis, K. Hartmann, Vet Clin Pathol 41, 558-567 (2012) doi:10.1111/j.1939-165X.2012.00464.X

59. A.H. Crawford, A.L. Stoll, D. Sanchez-Masian, J. Vet Intern Med. 31, 1477-1486 (2017) doi:10.1111/jvim.14791

60. M. Mwase, K. Shimada, C. Mumba et al., J. Comp. Pathol. 152, 265-268 (2015) doi:10.1016/j.jcpa.2014.12.006

61. M.R. Jinks, R.V. English, B.C. Gilger, Vet. Ophthalmol 19, 30-37 (2016) doi:10.1111/vop. 12324

62. S.J. Kim, V.G. Nguyen, Y.H. Park et al., Vaccines 8, 220 (2020) doi:10.3390/vaccines 8020220

63. B.G. Murphy, M. Perron, E. Murakami et al., Veterinary Microbiology 219, 226-233. (2018) doi:10.1016/j.vetmic.2018.04.026

64. N.C. Pedersen, Y. Kim, H. Liu et al., Journal of Feline Medicine and Surgery 20, 378392 (2018) doi:10.1177/1098612X17729626

65. N.C. Pedersen, M. Perron, M. Bannasch et al., Journal of Feline Medicine and Surgery 21, 271-281 (2019) doi:10.1177/1098612X19825701 
66. S. Paltrinieri, A. Giordano, A. Stranieri et al., Transboundary and Emerging Diseases (2020) doi:10.1111/tbed.13856

67. E. Porter, S. Tasker, M. J. Day et al., Vet. Res. 45, 49-59 (2014) doi:10.1186/12979716-45-49.

68. M. J. Day, M. C. Horzinek, R. D. Schultz et al., The Journal of Small Animal Practice 57, 1-45 (2016) doi:10.1111/jsap.2_12431

69. L. Sangl, K. Matiasek, S. Felten et al., J. Feline Med. Surg. 21, 133-142 (2019) doi:10.1177/1098612X18762883 